\title{
Applications of Mathematics
}

Ferdinand Chovanec; František Kôpka

Fuzzy equality and convergences for $F$-observables in $F$-quantum spaces

Applications of Mathematics, Vol. 36 (1991), No. 1, 32-45

Persistent URL: http://dml.cz/dmlcz/104442

\section{Terms of use:}

(C) Institute of Mathematics AS CR, 1991

Institute of Mathematics of the Czech Academy of Sciences provides access to digitized documents strictly for personal use. Each copy of any part of this document must contain these Terms of use.

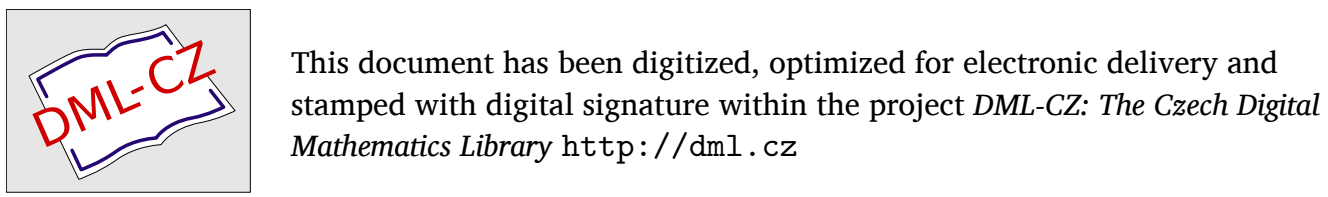




\title{
FUZZY EQUALITY AND CONVERGENCES FOR $F$-OBSERVABLES IN $F$-QUANTUM SPACES
}

\author{
Ferdinand Chovanec, František KôpKa
}

(Received July 27, 1989)

Summary. We introduce a fuzzy equality for $F$-observables on an $F$-quantum space which enables us to characterize different kinds of convergences, and to represent them by pointwise functions on an appropriate measurable space.

Keywords: $F$-quantum space, $F$-state, $F$-observable, representation theorem of $F$-observables, convergence of $F$-observables.

AMS Classification: 28A20.

Let $(\Omega, \mathscr{S}, E)$ be a probability space and $f: \Omega \rightarrow R^{1}$ a real valued, $\mathscr{S}$-measurable random variable, i.e. $f^{-1}(E) \in \mathscr{S}$ for any set $E \in B\left(R^{1}\right)$, where $B\left(R^{1}\right)$ is the Borel $\sigma$-algebra of the real line $R^{1}$. The mapping $x: B\left(R^{1}\right) \rightarrow \mathscr{S}$ defined as $x(E)=f^{-1}(E)$, $E \in B\left(R^{1}\right)$, is a $\sigma$-homomorphism, called an observable of $\mathscr{S}$.

Gudder and Mullikin [1] introduced many types of convergences for the observables in a quantum logic. Motivated by their definitions, we present some convergences of $F$-observables in $F$-quantum spaces.

\section{F-QUANTUM SPACE}

We recall that according to [2], an $F$-quantum space is a couple $(\Omega, M)$, where $\Omega$ is a nonvoid set and $M \subset[0,1]^{\Omega}$ is a system of fuzzy subsets of $\Omega$ such that

(i) if $1(\omega)=1$ for any $\omega \in \Omega$, then $1 \in M$,

(ii) $a \in M$ implies $a^{\perp}:=1-a \in M$,

(iii) if $1 / 2(\omega)=1 / 2$ for any $\omega \in \Omega$, then $1 / 2 \notin M$,

(iv) if $\left\{a_{n}\right\}_{n \in N} \subset M$, then $\bigcup_{n \in N} a_{n}:=\sup _{n \in N} a_{n} \in M$. The $\bigcap$ is a fuzzy union, and the fuzzy intersection, $\bigcap$, is defined via $\bigcap_{n \in N} a_{n}:=\inf _{n \in N} a_{n}$.
The set $M$ is also called a soft fuzzy $\sigma$-algebra [4].

The soft fuzzy $\sigma$-algebra $M$ can be regarded as a partially ordered set in which we define $a \leqq b$ iff $a(\omega) \leqq b(\omega)$ for any $\omega \in \Omega$. 
Using the complementation $\perp: a \mapsto a^{\perp}=1-a$, we see that it satisfies two conditions:

(i) $\left(a^{\perp}\right)^{\perp}=a$ for any $a \in M$,

(ii) $a \leqq b$ implies $b^{\perp} \leqq a^{\perp}$.

Two fuzzy sets $a$ and $b$ are called orthogonal or $W$-separated and we write $a \perp b$, iff $a \leqq b^{\perp}$.

It is clear that $a \perp a^{\perp}$ for any $a \in M$.

We say that a fuzzy set $a \in M$ is a $W$-empty set ( $W$-universum), iff $a \leqq a^{\perp}\left(a \geqq a^{\perp}\right)$. It is evident that the following assertions are equivalent:

(i) $a$ is a $W$-empty set ( $W$-universum),

(ii) $a \leqq 1 / 2(a \geqq 1 / 2)$,

(iii) $a \cap a^{\perp}=a\left(a \cup a^{\perp}=a\right)$,

(iv) $a^{\perp}$ is a $W$-universum ( $W$-empty set).

We denote by $W_{0}(M)$ and $W_{1}(M)$ the sets of all $W$-empty sets and $W$-universes, respectively, from $M$.

If $a, b \in M, a \leqq b, b \in W_{0}(M)$ then $a \in W_{0}(M)$ and if $a \leqq b, a \in W_{1}(M)$ then $b \in W_{1}(M)$.

Let $B\left(R^{1}\right)$ be the Borel $\sigma$-algebra of the real line $R^{1}$. By an $F$-observable of $(\Omega, M)$ we mean a mapping $x: B\left(R^{1}\right) \rightarrow M$ such that

(i) $x\left(E^{c}\right)=x(E)^{\perp}, E \in B\left(R^{1}\right), E^{c}=R^{1}-E$,

(ii) $x(E) \perp x(F)$ if $E \cap F=\emptyset, E, F \in B\left(R^{1}\right)$,

(iii) if $\left\{E_{n}\right\}_{n \in N} \subset B\left(R^{1}\right), E_{i} \cap E_{j}=\emptyset$ for $i \neq j$, then $x\left(\bigcup_{n \in N} E_{n}\right)=\bigcup_{n \in N} x\left(E_{n}\right)$.

If $a$ is a fuzzy set from $M$, then the mapping $x_{a}$ defined via

$$
x_{a}(E)= \begin{cases}a \cap a^{\perp} & \text { if } 0,1 \notin E, \\ a^{\perp} & \text { if } 0 \in E, 1 \notin E, \\ a & \text { if } 0 \notin E, 1 \in E, \\ a \cup a^{\perp} & \text { if } 0,1 \in E\end{cases}
$$

for any $E \in B\left(R^{1}\right)$ is an $F$-observable of $(\Omega, M)$ and plays the role of the indicator of the fuzzy event $a \in M$.

If $x$ is an $F$-observable and $f: R^{1} \rightarrow R^{1}$ is a Borel function, then $f \circ x: E \mapsto$ $\mapsto x\left(f^{-1}(E)\right), E \in B\left(R^{1}\right)$, is an $F$-observable of $(\Omega, M)$, too. In particular, if $f(t)=|t|$, $t \in R^{1}$, we put $|x|=f \circ x$, etc. Similarly $-x$ is an $F$-observable defined via

$$
-x(E)=x(\{t:-t \in E\}) \text { for any } E \in B\left(R^{1}\right) .
$$

Let $x$ and $y$ be two $F$-observables. By the sum of $x$ and $y$ (see [5]) we mean an $F$-observable $z$ such that

$$
z((-\infty, t))=\bigcup_{r \in Q} x((-\infty, r)) \cap y((-\infty, t-r))
$$


for any $t \in R^{1}$, where $Q$ is the set of all rationals in the real line $R^{1}$ and we write $z=x+y$. In the paper [5] it has been proved that the sum of any pair of $F$ observables exists and is unique. We shall denote by $O(M)$ the set of all $F$-observables of $(\Omega, M)$.

An $F$-state on $(\Omega, M)$ is a mapping $m: M \rightarrow[0,1]$ such that

(i) $m\left(a \cup a^{\perp}\right)=1$ for any $a \in M$,

(ii) if $\left\{a_{n}\right\}_{n \in N} \subset M, a_{i} \leqq a_{j}^{\perp}$ for $i \neq j$, then $m\left(\bigcup_{n \in N} a_{n}\right)=\sum_{n \in N} m\left(a_{n}\right)$.

According to [6], we define $K(M)$ as the set of all subsets $A \subset \Omega$ such that there is a fuzzy set $a \in M$ satisfying

$$
\{a>1 / 2\} \subset A \subset\{a \geqq 1 / 2\},
$$

where $\{a>1 / 2\}=\{\omega \in \Omega: a(\omega)>1 / 2\}$, similarly for $\{a \geqq 1 / 2\}$.

The following result holds (see [6], [7]).

Theorem 1.1. Let $(\Omega, M)$ be an F-quantum space. Then $K(M)$ is a $\sigma$-algebra of subsets of the set $\Omega$. If $m$ is an $F$-state, the function $P=P_{m}: K(M) \rightarrow[0,1]$ defined via

$$
P(A)=m(a), \quad A \in K(M),
$$

where $A$ and a satisfy (1.3), is a probability measure on $K(M)$ with

$$
P(\{a=1 / 2\})=0 \text { for any } a \in M .
$$

Moreover, if $m, n$ are $F$-states such that $m \neq n$, then $P_{m} \neq P_{n}$.

Conversely, let $P$ be any probability measure on $K(M)$ with (1.5), then the mapping $m=m_{P}: M \rightarrow[0,1]$ defined via

$$
m(a)=P(A), \quad a \in M,
$$

where $a$ and $A$ fulfil (1.3), is an F-state. Moreover, if $P \neq Q$, then $m_{P} \neq m_{Q}$. In addition, $m=m_{P_{m}}$ and $P=P_{m_{P}}$.

Lemma 1.2. Let $\left\{a_{n}\right\}_{n \in N} \subset M$. Then

(i) $\bigcup_{n \in N}\left\{a_{n} \geqq 1 / 2\right\} \subset\left\{\bigcup_{n \in N} a_{n} \geqq 1 / 2\right\}$,

(ii) $\bigcup_{n \in N}\left\{a_{n}>1 / 2\right\}=\left\{\bigcup_{n \in N} a_{n}>1 / 2\right\}$,

(iii) $\left\{\bigcap_{n \in N} a_{n}>1 / 2\right\} \subset \bigcap_{n \in N}\left\{a_{n}>1 / 2\right\}$,

(iv) $\left\{\bigcap_{n \in N} a_{n} \geqq 1 / 2\right\}=\bigcap_{n \in N}\{a \geqq 1 / 2\}$,

(v) if $a_{n} \in W_{1}(M)$ for any $n \in N$, then

$$
\bigcup_{n \in N}\left\{a_{n}=1 / 2\right\} \subset\left\{\bigcap_{n \in N} a_{n}=1 / 2\right\} .
$$


Proof. It is straightforward and, therefore, it is omitted.

A. Dvurečenskij proved the following representation theorem.

Theorem 1.3 [7]. For any $x \in O(M)$ there is a $K(M)$-measurable, real-valued function $f$ on $\Omega$ such that

$$
\{x(E)>1 / 2\} \subset f^{-1}(E) \subset\{x(E) \geqq 1 / 2\}
$$

for any $E \in B\left(R^{1}\right)$. If $g$ is any $K(M)$-measurable, real-valued function on $\Omega$ satisfying (1.7), then

$$
\{\omega \in \Omega: f(\omega) \neq g(\omega)\} \subset\{x(\emptyset)=1 / 2\} .
$$

Conversely, let $f: \Omega \rightarrow R^{1}$ be any $K(M)$-measurable function. Then there is an F-observable $x$ satisfying (1.7). If $y$ is any F-observable satisfying (1.7), then $x(E) \cap y\left(E^{c}\right) \in W_{0}(M)$ for any $E \in B\left(R^{1}\right)$.

We shall denote by $F(M)$ the set of all $K(M)$-measurable real-valued functions on $\Omega$ and write $x \sim f$ for $x \in O(M)$ and $f \in F(M)$ such that (1.7) holds.

Theorem 1.4 [7]. Let $x \sim f, y \sim g$ and $h$ be any Borel function. Then

(i) $x+y \sim f+g$,

(ii) $h \circ x \sim h \circ f$,

(iii) $x \cdot y \sim f . g$, where $x \cdot y:=1 / 2\left((x+y)^{2}-x^{2}-y^{2}\right)$,

(iv) if $f \geqq 0$ then $x([0, \infty))=x\left(R^{1}\right)$.

Lemma 1.5. Let $a \in M, A \in K(M)$ be such that the condition (1.3) holds. Then $x_{a} \sim I_{A}$, where $I_{A}$ is the indicator of the set $A$.

Proof. By the assumptions of the lemma, $\{a>1 / 2\} \subset A \subset\{a \geqq 1 / 2\}$. Let $E \in B\left(R^{1}\right)$ and $0,1 \notin E$. Then $x_{a}(E)=a \cap a^{\perp}$ and $\left\{x_{a}(E)>1 / 2\right\}=\left\{a \cap a^{\perp}>1 / 2\right\}=$ $=\emptyset=I_{A}^{-1}(E) \subset\left\{x_{a}(E) \geqq 1 / 2\right\}$. If $E \in B\left(R^{1}\right)$ is such that $0 \in E$ and $1 \notin E$, then $x_{a}(E)=a^{\perp}$ and $\left\{x_{a}(E)>1 / 2\right\}=\left\{a^{\perp}>1 / 2\right\} \subset A^{c}=I_{A}^{-1}(E) \subset\left\{a^{\perp} \geqq 1 / 2\right\}=$ $=\left\{x_{a}(E) \geqq 1 / 2\right\}$. If $E \in B\left(R^{1}\right)$ is such that $0,1 \in E$, then $x_{a}(E)=a \cup a^{\perp}$, and due to Lemma 1.2, $\left\{x_{a}(E)>1 / 2\right\}=\left\{a \cup a^{\perp}>1 / 2\right\} \subset A \cup A^{c}=\Omega=I_{A}^{-1}(E)=$ $=\left\{a \cup a^{\perp} \geqq 1 / 2\right\}=\left\{x_{a}(E) \geqq 1 / 2\right\}$.

Finally, if $E \in B\left(R^{1}\right)$ is such that $0 \notin E$ and $1 \in E$, then $x_{a}(E)=a$ and $I_{A}^{-1}(E)=A$.

We see that $\left\{x_{a}(E)>1 / 2\right\} \subset I_{A}^{-1}(E) \subset\left\{x_{a}(E) \geqq 1 / 2\right\}$ for any $E \in B\left(R^{1}\right)$, which implies $x_{a} \sim I_{A}$.

\section{FUZZY EQUALITIES AND FUZZY INEQUALITIES}

Let $(\Omega, M)$ be an $F$-quantum space. According to [8], a non-void subset $I$ of $M$ is said to be an $F$-ideal $(F-\sigma$-ideal $)$ if: 
(i) $a \cap a^{\perp} \in I$ for any $a \in M$,

(ii) if $a \in M$ and $a \leqq b, b \in I$, then $a \in I$,

(iii) if $a \cap b \in I$ for some $b \in W_{1}(M)$, then $a \in I$,

(iv) $a, b \in I$ implies $a \cup b \in I\left(\bigcup_{n \in N} a_{n} \in I\right.$ whenever $\left.\left\{a_{n}\right\}_{n \in N} \subset I\right)$.

Suppose that $I$ is an $F$ - $\sigma$-ideal and put $a \sim_{1} b$ iff $a \cap b^{\perp}$ and $a^{\perp} \cap b$ are from $I$. Then $\sim_{I}$ is a congruence $(\sigma$-)relation on $M$ (see [8]), i.e.,

(i) $\sim_{I}$ is an equivalence relation on $M$,

(ii) $a \cap a^{\perp} \sim_{I} 0$ for any $a \in M$,

(iii) $a \sim_{I} b$ implies $a^{\perp} \sim_{I} b^{\perp}$,

(iv) $a_{1} \sim_{I} b_{1}$ and $a_{2} \sim_{I} b_{2}$ imply $a_{1} \cup a_{2} \sim_{I} b_{1} \cup b_{2}$ $\left(a_{n} \sim_{I} b_{n}, n \in N\right.$, imply $\left.\bigcup_{n \in N} a_{n} \sim_{I} \bigcup_{n \in N} b_{n}\right)$.

Denote by

$$
I_{0}=\left\{a \in M: \text { there is } a c \in W_{1}(M) \text { such that } a \cap c \in W_{0}(M)\right\} .
$$

Then $I_{0}$ is an $F$ - $\sigma$-ideal, $I_{0} \subset I$ for any $F$ - $\sigma$-ideal and $1 \notin I_{0}$. In particular, if $M$ consists exclusively from crisp subsets of $\Omega$, then $I_{0}=\{\emptyset\}$.

Definition 2.1. We say that two fuzzy sets $a, b \in M$ are fuzzy equal and we write $a={ }_{p} b$, iff $a \cap b^{\perp}, a^{\perp} \cap b \in I_{0}$.

Let $x, y \in O(M)$. We say that $x$ and $y$ are fuzzy equal and we write $x=_{F} y$, iff $x(E) \cap y\left(E^{c}\right) \in I_{0}$ for every $E \in B\left(R^{1}\right)$.

Let $A, B \in K(M)$. We say that $A$ and $B$ are fuzzy equal and we write $A={ }_{F} B$, iff there is a $c \in W_{1}(M)$ such that $A \triangle B \subset\{\omega \in \Omega: c(\omega)=1 / 2\}$, where $A \triangle B=$ $=A \cap B^{c} \cup A^{c} \cap B$.

Let $f, g: \Omega \rightarrow R^{1}$ be $K(M)$-measurable functions. We say that $f$ and $g$ are fuzzy equal and write $f={ }_{F} g$, iff there is a $c \in W_{1}(M)$ such that $\{\omega \in \Omega: f(\omega) \neq g(\omega)\} \subset$ $\subset\{\omega \in \Omega: c(\omega)=1 / 2\}$.

The relation $=_{P}$ is an equivalence relation on $M, O(M), K(M)$ and $F(M)$. It is simple to verify that the following assertions hold:

(i) $a \cap a^{\perp}=_{F} 0$ and $a \cup a^{\perp}=_{F} 1$ for any $a \in M$,

(ii) $x(\emptyset)={ }_{F} 0$ and $x\left(R^{1}\right)={ }_{F} 1$ for any $x \in O(M)$.

Lemma 2.2. Let $a, b \in M$ and let $A, B \in K(M)$ be such that (1.3) holds. Then

(i) $a={ }_{F} b$ if and only if $A={ }_{F} B$,

(ii) $a={ }_{F} b$ implies $m(a)=m(b)$, where $m$ is an F-state on $M$.

Proof. (i) If $a={ }_{F} b$, then there are $c, d \in W_{1}(M)$ such that $a \cap b^{\perp} \cap c \in W_{0}(M)$ and $a^{\perp} \cap b \cap d \in W_{0}(M)$, which implies $\left\{a \cap b^{\perp}>1 / 2\right\} \subset\{c=1 / 2\}$ and $\left\{a^{\perp} \cap b>1 / 2\right\} \subset\{d=1 / 2\}$. From (1.3) we have $\{a>1 / 2\} \subset A \subset\{a \geqq 1 / 2\}$ and $\left\{b^{\perp}>1 / 2\right\} \subset B^{c} \subset\left\{b^{\perp} \geqq 1 / 2\right\}$, which gives, by Lemma $1.2,\left\{a \cap b^{\perp}>1 / 2\right\} \subset$ 
$\subset A \cap B^{c} \subset\left\{a \cap b^{\perp} \geqq 1 / 2\right\}=\left\{a \cap b^{\perp}>1 / 2\right\} \cup\left\{a \cap b^{\perp}=1 / 2\right\} \subset\{c=1 / 2\} \cup$ $\cup\left\{a \cap b^{\perp} \cup\left(a \cap b^{\perp}\right)^{\perp}=1 / 2\right\}=\left\{c \cap\left(\left(a \cap b^{\perp}\right) \cup\left(a \cap b^{\perp}\right)^{\perp}\right)=1 / 2\right\}$. Put $u=c \cap$ $\cap\left(\left(a \cap b^{\perp}\right) \cup\left(a \cap b^{\perp}\right)^{\perp}\right)$. Then $u \in W_{1}(M)$ and $A \cap B^{c} \subset\{u=1 / 2\}$. Similarly $A^{c} \cap B \subset\{v=1 / 2\}$, where $v=d \cap\left(\left(a^{\perp} \cap b\right) \cup\left(a^{\perp} \cap b\right)^{\perp}\right) \in W_{1}(M)$. Therefore, $A \triangle B=A \cap B^{c} \cup A^{c} \cap B \subset\{u=1 / 2\} \cup\{v=1 / 2\}=\{u \cap v=1 / 2\}$, where $u \cap$ $\cap v \in W_{1}(M)$. which gives $A={ }_{F} B$.

Let now $A={ }_{F} B$. Then there is a $c \in W_{1}(M)$ such that $A \cap B^{c} \cup A^{c} \cap B \subset$ $\subset\{c=1 / 2\}$. We have $\left\{a \cap b^{\perp}>1 / 2\right\} \subset A \cap B^{c} \subset\{c=1 / 2\}$ and $\left\{a^{\perp} \cap b>1 / 2\right\} \subset$ $\subset A^{c} \cap B \subset\{c=1 / 2\}$ and this is equivalent to $a \cap b^{\perp} \cap c \leqq 1 / 2$ and $a^{\perp} \cap b \cap$ $\cap c \leqq 1 / 2$.

(ii) If $a={ }_{F} b$ then from (i) we have $A={ }_{F} B$, which implies that there is a $c \in W_{1}(M)$ such that $A \triangle B \subset\{c=1 / 2\}$. Let $P$ be a probability measure on $K(M)$ defined via (1.4). From (1.5) we have $P\left(A \cap B^{c}\right)=0$ and $P\left(A^{c} \cap B\right)=0$. Then $P(A)=$ $=P\left(A \cap B^{c} \cup A \cap B\right)=P\left(A \cap B^{c}\right)+P(A \cap B)=P(A \cap B)$ and similarly $P(B)=$ $=P(A \cap B)$, which gives the equality $P(A)=P(B)$ and by $(1.6) m(a)=m(b)$.

Lemma 2.3. Let $x_{a}$ and $x_{b}$ be two indicators of fuzzy sets $a$ and $b$, respectively. Then $x_{a}={ }_{F} x_{b}$ if and only if $a={ }_{F} b$.

Proof. It is evident.

Lemma 2.4. Let $x, y \in O(M)$. Then $x=_{F} y$ if and only if there is a $c \in W_{1}(M)$ such that $x(E) \cap y\left(E^{c}\right) \cap c \in W_{0}(M)$ for any $E \in B\left(R^{1}\right)$.

Proof. Let $\left\{E_{n}\right\}_{n \in N}$ be a generator of $B\left(R^{1}\right)$. If $x=_{F} y$, then there are $u_{n}, v_{n} \geqq 1 / 2$ such that $x\left(E_{n}\right) \cap y\left(E_{n}^{c}\right) \cap u_{n} \leqq 1 / 2$ and $x\left(E_{n}^{c}\right) \cap y\left(E_{n}\right) \cap v_{n} \leqq 1 / 2$ for any $n \in N$. Denote $c_{n}=u_{n} \cap v_{n}$ and put $c=\bigcap_{n \in N} c_{n}$. Then $c \geqq 1 / 2$ and $x\left(E_{n}\right) \cap y\left(E_{n}^{c}\right) \cap c \leqq$ $\leqq x\left(E_{n}\right) \cap y\left(E_{n}^{c}\right) \cap c_{n} \leqq x\left(E_{n}\right) \cap y\left(E_{n}^{c}\right) \cap u_{n} \leqq 1 / 2$ and similarly $x\left(E_{n}^{c}\right) \cap y\left(E_{n}\right) \cap c \leqq$ $\leqq 1 / 2$. Denote

$$
K=\left\{E \in B\left(R^{1}\right): x(E) \cap y\left(E^{c}\right) \cap c \leqq 1 / 2, x\left(E^{c}\right) \cap y(E) \cap c \leqq 1 / 2\right\} .
$$

The system $K$ is a non-empty set containing the generator $\left\{E_{n}\right\}_{n \in N}$. Moreover, $x(\emptyset) \cap$ $\cap y\left(R^{1}\right) \cap c=x(\emptyset) \leqq 1 / 2$ and $x\left(R^{1}\right) \cap y(\emptyset) \cap c=y(\emptyset) \leqq 1 / 2$ imply that $\emptyset \in K$ and $R^{1} \in K$. If $\left\{A_{n}\right\}_{n \in N} \subset K$, then $x\left(\bigcup_{n \in N} A_{n}\right) \cap y\left(\bigcap_{n \in N} A_{n}^{c}\right) \cap c=\bigcup_{n \in N} x\left(A_{n}\right) \cap \bigcap_{n \in N} y\left(A_{n}^{c}\right) \cap c \leqq$ $\leqq \bigcup_{n \in N} x\left(A_{n}\right) \cap y\left(A_{n}^{c}\right) \cap c \leqq 1 / 2$, therefore, $\bigcup_{n \in N} A_{n} \in K$. We have proved that $K$ is a $\sigma$-algebra, consequently, $K=B\left(R^{1}\right)$.

The converse assertion is obvious.

Proposition 2.5. Let $x, y \in O(M)$ and $f, g \in F(M)$ be such that $x \sim f, y \sim g$. The following statements are equivalent:

(i) $x={ }_{F} y$.

(ii) $f={ }_{F} g$. 
Proof. Suppose that (i) holds. From $x \sim f, y \sim g$ we have $\{x(E)>1 / 2\} \subset$ $\subset f^{-1}(E) \subset\{x(E) \geqq 1 / 2\},\left\{y\left(E^{c}\right)>1 / 2\right\} \subset g^{-1}\left(E^{c}\right) \subset\left\{y\left(E^{c}\right) \geqq 1 / 2\right\}$ and $\left\{x(E) \cap y\left(E^{c}\right)>1 / 2\right\} \subset f^{-1}(E) \cap g^{-1}\left(E^{c}\right) \subset\left\{x(E) \cap y\left(E^{c}\right) \geqq 1 / 2\right\}$, too. By Lemma 2.4 , there is a $c \geqq 1 / 2$ such that $x(E) \cap y\left(E^{c}\right) \cap c \leqq 1 / 2$ for any $E \in B\left(R^{1}\right)$, which gives $\left\{x(E) \cap y\left(E^{c}\right)>1 / 2\right\} \subset\{c=1 / 2\}$ for any $E \in B\left(R^{1}\right)$. Since $\{x(E)=1 / 2\}=$ $=\left\{x\left(R^{1}\right)=1 / 2\right\}$ and $\left\{y\left(E^{c}\right)=1 / 2\right\}=\left\{y\left(R^{1}\right)=1 / 2\right\}$, we have $\left\{x(E) \cap y\left(E^{c}\right) \geqq\right.$ $\geqq 1 / 2\}=\left\{x(E) \cap y\left(E^{c}\right)>1 / 2\right\} \cup\left\{x(E) \cap y\left(E^{c}\right)=1 / 2\right\} \subset\{c=1 / 2\} \cup$ $\cup\left\{x\left(R^{1}\right) \cap y\left(R^{1}\right)=1 / 2\right\}=\left\{c \cap x\left(R^{1}\right) \cap y\left(R^{1}\right)=1 / 2\right\}$ for any $E \in B\left(R^{1}\right)$. Put $d=c \cap x\left(R^{1}\right) \cap y\left(R^{1}\right)$, then $d \in W_{1}(M)$ and we have $f^{-1}(E) \cap g^{-1}\left(E^{c}\right) \subset$ $\left.\subset x(E) \cap y\left(E^{c}\right) \geqq 1 / 2\right\} \subset\{d=1 / 2\}$ for any $E \in B\left(R^{1}\right)$, which implies $f^{-1}(E) \cap$ $\cap g^{-1}\left(E^{c}\right) \cup f^{-1}\left(E^{c}\right) \cap g^{-1}(E) \subset\{d=1 / 2\}$, too.

Finally, $\quad\{\omega \in \Omega: f(\omega) \neq g(\omega)\}=\bigcup_{r \in Q}(\{\omega: f(\omega)<r \leqq g(\omega)\} \cup\{\omega: g(\omega)<r \leqq$ $\leqq f(\omega)\})=\bigcup_{r \in Q}\left(f^{-1}((-\infty, r)) \cap g^{-1}\left((-\infty, r)^{c}\right) \cup f^{-1}\left((-\infty, r)^{c}\right) \cap g^{-1}((-\infty, r))\right) \subset$ $\subset\{d=1 / 2\}$, where $Q$ is the set of all rationals in the real line, and this gives $f={ }_{F} g$.

Suppose now that (ii) holds. By definition, there is a $c \in W_{1}(M)$ such that $\{\omega: f(\omega) \neq g(\omega)\} \subset\{\omega: c(\omega)=1 / 2\}$. For any $E \in B\left(R^{1}\right)$ we have $\left\{x(E) \cap y\left(E^{c}\right)>\right.$ $>1 / 2\} \subset f^{-1}(E) \cap g^{-1}\left(E^{c}\right) \subset\{\omega: f(\omega) \neq g(\omega)\} \subset\{\omega: c(\omega)=1 / 2\}$, which implies $x(E) \cap y\left(E^{c}\right) \cap c \leqq 1 / 2$.

Corollary 2.6. Let $x, y \in O(M)$ and $f, g \in F(M)$ be such that $x \sim f, y \sim g$. Let $m$ be an F-state on $M$ and let $P$ be the probability measure on $K(M)$ defined via (1.4). If $x={ }_{F} y$ then $f=g$ almost everywhere with respect to the measure $P$, i.e. $P(\{\omega: f(\omega) \neq g(\omega)\})=0$.

We define a mapping $o: B\left(R^{1}\right) \rightarrow M$ via

$$
o(E)=\left\{\begin{array}{lll}
1 & \text { if } & 0 \in E, \\
0 & \text { if } & 0 \notin E
\end{array}\right.
$$

for any $E \in B\left(R^{1}\right)$. The mapping $o$ is an $F$-observable of $M$. Moreover, if $f_{0}(\omega)=0$ for any $\omega \in \Omega$, then $f_{0}$ is $K(M)$-measurable real-valued function from $\Omega$ into the real line $R^{1}$ and $o \sim f_{0}$.

Lemma 2.7. Let $f, g \in F(M)$. Then $f={ }_{F} g$ if and only if $f-g={ }_{F} 0$.

Proof. If $f={ }_{F} g$ then there is a $c \in W_{1}(M)$ such that $\{\omega: f(\omega) \neq g(\omega)\} \subset$ $\subset\{c=1 / 2\}$. But $\{\omega:(f-g)(\omega) \neq 0\}=\{\omega: f(\omega) \neq g(\omega)\} \subset\{c=1 / 2\}$, which implies $f-g={ }_{F} 0$.

The converse assertion is evident.

Proposition 2.8. Let $x, y \in O(M)$. Then $x={ }_{F} y$ if and only if $x-y={ }_{F} o$.

Proof. It follows from Lemma 2.7 and Proposition 2.5. 
Proposition 2.9. Let $x, y \in O(M)$. The following statements are equivalent:

(i) $x={ }_{F} y$,

(ii) $(x-y)(\{0\})={ }_{F} 1$.

Proof. Let (i) hold and let $f, g \in F(M)$ be such that $x \sim f, y \sim g$. By Proposition 2.5, $f={ }_{F} g$ and by Lemma 2.7, $f-g={ }_{F} 0$, which is equivalent to $x-y={ }_{F} o$. Lemma 2.4 yields $(x-y)(\{0\})={ }_{F} o(\{0\})=1$.

Suppose now that (ii) holds. Then there is a $c \in W_{1}(M)$ such that $(x-y)\left(\{0\}^{c}\right) \cap$ $\cap 1 \cap c \leqq 1 / 2$, which implies $\left\{(x-y)\left(\{0\}^{c}\right)>1 / 2\right\} \subset\{c=1 / 2\}$. If $E \in B\left(R^{1}\right)$, $0 \notin E$, then $E \subset\{0\}^{c}$ and $(x-y)(E) \leqq(x-y)\left(\{0\}^{c}\right)$ and so $\{(x-y)(E)>1 / 2\} \subset$ $\subset\left\{(x-y)\left(\{0)^{c}\right)>1 / 2\right\} \subset\{c=1 / 2\}$, therefore $(x-y)(E)={ }_{F} 0=o(E)$ whenever $0 \notin E$. If $0 \in E$, then $0 \notin E^{c}$ and $(x-y)\left(E^{c}\right)={ }_{F} 0=o\left(E^{c}\right)$, which implies $(x-y)$. $.(E)={ }_{F} 1=o(E)$ whenever $0 \in E$. We have proved that $(x-y)(E)={ }_{F} o(E)$ for any $E \in B\left(R^{1}\right)$, which is equivalent to $(x-y)={ }_{F} o$ as well as to $x={ }_{F} y$.

Definition 2.10. We say that a fuzzy set $a \in M$ is fuzzy less or equal to $b \in M$ and write $a \leqq_{F} b$, iff $a \cap b={ }_{F} a$.

Let $x, y \in O(M)$. We say that $x$ is fuzzy less or equal to $y$ and write $x \leqq_{F} y$, iff $(y-x)([0, \infty))={ }_{F} 1$.

Let $A, B \in K(M)$. We say that $A$ is a fuzzy subset of $B$ and write $A \subset{ }_{F} B$, iff $A \cap B={ }_{F} A$.

Let $f, g \in F(M)$. We say that $f$ is fuzzy less or equal to $g$ and write $f \leqq_{F} g$, iff there is a $c \in W_{1}(M)$ such that $\{\omega: f(\omega)>g(\omega)\} \subset\{c=1 / 2\}$.

The sets $M, O(M), K(M), F(M)$ are sets partially ordered by the relation $\leqq_{F}$.

Lemma 2.11. Let $a, b \in M$ and $A, B \in K(M)$ be such that (1.3) holds. Let $m$ be an $F$-state. Then the following assertion hold:

(i) $a \leqq_{F} b$ if and only if $A \subset_{F} B$.

(ii) If $a \leqq_{F} b$, then $m(a) \leqq m(b)$.

(iii) If $a \leqq_{F} b^{\perp}$, then $m(a \cup b)=m(a)+m(b)$.

Proof. (i) It is evident.

(ii) If $a \leqq_{F} b$ then by (i) $A \subset_{F} B$, which implies $A \cap B={ }_{F} A$ and Lemma 2.2 yields $P(A \cap B)=P(A)$. Then we have $m(b)=P(B)=P\left(A \cap B \cup A^{c} \cap B\right)=$ $=P(A \cap B)+P\left(A^{c} \cap B\right)=P(A)+P\left(A^{c} \cap B\right) \geqq P(A)=m(a)$.

(iii) By (ii) we have $P\left(A \cap B^{c}\right)=P(A)$ and then $m(a \cup b)=P(A \cup B)=$ $=P\left(A \cap B^{c}\right)+P(B)=P(A)+P(B)=m(a)+m(b)$.

Proposition 2.12. Let $x, y \in O(M)$ and $f, g \in F(M)$ be such that $x \sim f, y \sim g$. Then $x \leqq_{F} y$ if and only if $f \leqq_{F} g$.

Proof. If $x \leqq_{F} y$ then from definition $(y-x)([0, \infty))={ }_{F} 1$, which implies the existence of a $c \in W_{1}(M)$ such that $(y-x)([0, \infty))^{\perp} \cap c \leqq 1 / 2$ and $\{(y-x)$. $.((-\infty, 0))>1 / 2\} \subset\{c=1 / 2\}$. The assumptions of the proposition and (i) of 
Theorem 1.4 give $y-x \sim g-f$, and from Theorem 1.3 we have

$$
\begin{aligned}
& \{(y-x)((-\infty, 0))>1 / 2\} \subset(g-f)^{-1}((-\infty, 0) \subset \\
& \subset\{(y-x)((-\infty, 0)) \geqq 1 / 2\}=\{(y-x)((-\infty, 0))>1 / 2\} \cup \\
& \cup\{(y-x)((-\infty, 0))=1 / 2\} \subset\{c=1 / 2\} \cup \\
& \cup\left\{(y-x)\left(R^{1}\right)=1 / 2\right\}=\left\{c \cap(y-x)\left(R^{1}\right)=1 / 2\right\} .
\end{aligned}
$$

Put $d=c \cap(y-x)\left(R^{1}\right)$, then $d \in W_{1}(M)$ and $\{\omega: f(\omega)>g(\omega)\}=\{\omega:(g-f)$. . $(\omega)<0\}=(g-f)^{-1}((-\infty, 0)) \subset\{d=1 / 2\}$, which implies $f \leqq_{F} g$.

Suppose now that $f \leqq_{F} g$. Then there is a $c \in W_{1}(M)$ such that $\{\omega: f(\omega)>g(\omega)\}=$ $=(g-f)^{-1}((-\infty, 0)) \subset\{c=1 / 2\}$. From Theorem 1.3 we have $\{(y-x)$. $.((-\infty, 0))>1 / 2\} \subset(g-f)^{-1}((-\infty, 0)) \subset\{c=1 / 2\}=\left\{c \cup c^{\perp}=1 / 2\right\}$. Put $d=$ $=c \cup c^{\perp}$. It is evident that $d \in W_{1}(M)$ and $\{(y-x)((-\infty, 0))>1 / 2\} \subset\{d=1 / 2\}$, therefore, $(y-x)((-\infty, 0)) \cap d \leqq 1 / 2$ and also $(y-x)([0, \infty)) \cap 1^{\perp} \cap d=0 \leqq$ $\leqq 1 / 2$, which implies $(y-x)([0, \infty))={ }_{F} 1$.

\section{CONVERGENCES OF F-OBSERVABLES}

Let $x$ be an $F$-observable. Then the mean value of $x$ in an $F$-state $m$ is the expression $m(x)$ defined by

$$
m(x)=\int_{R^{1}} t \mathrm{~d} m_{x}(t),
$$

(if the right-hand side exists and is finite), where $m_{x}$ is a probability measure on $B\left(R^{1}\right)$ defined via $m_{x}(E)=m(x(E)), E \in B\left(R^{1}\right)$, and we say that the $F$-observable $x$ is integrable and write $m(x)=\int x \mathrm{~d} m$. Moreover, if $f$ is a Borel measurable function, then $m(f \circ x)=\int_{R^{1}} f(t) \mathrm{d} m_{x}(t)$, in the sense that if one side exists, then the other exists, and both are equal.

Motivated by many types of convergences for the observables in quantum logics [1] we introduce the following notions.

Definition 3.1. We say that a sequence $\left\{x_{n}\right\}_{n \in N} \subset O(M)\left(\left\{f_{n}\right\}_{n \in N} \subset F(M)\right)$ converges to $x \in O(M)(f \in F(M))$ :

(1) fuzzy everywhere, if, for every $\varepsilon>0$,

$$
\bigcup_{k=1}^{\infty} \bigcap_{n=k}^{\infty}\left(x-x_{n}\right)([-\varepsilon, \varepsilon])={ }_{F} 1\left(\bigcup_{k=1}^{\infty} \bigcap_{n=k}^{\infty}\left(f-f_{n}\right)^{-1}([-\varepsilon, \varepsilon])={ }_{F} \Omega\right.
$$

(2) almost everywhere in an F-state $m$ (in a measure $P$ ), if, for every $\varepsilon>0$,

$$
\begin{aligned}
& m\left(\bigcup_{k=1}^{\infty} \bigcap_{n=k}^{\infty}\left(x-x_{n}\right)([-\varepsilon, \varepsilon])\right)=1 \\
& \left(P\left(\bigcup_{k=1}^{\infty} \bigcap_{n=k}^{\infty}\left(f-f_{n}\right)^{-1}([-\varepsilon, \varepsilon])\right)=1\right) ;
\end{aligned}
$$


(3) fuzzy uniformly, if, for every $\varepsilon>0$, there is an integer $k$ such that $\left(x-x_{n}\right)$. $\cdot([-\varepsilon, \varepsilon])={ }_{F} 1$

$$
\left(\left(f-f_{n}\right)^{-1}([-\varepsilon, \varepsilon])={ }_{F} \Omega\right) \text { for all } n \geqq k ;
$$

(4) uniformly almost everywhere in an F-state $m$ (in a measure P), if, for every $\varepsilon>0$, there is an integer $k$ such that $m\left(\left(x-x_{n}\right)([-\varepsilon, \varepsilon])\right)=1$ $\left(P\left(\left(f-f_{n}\right)^{-1}([-\varepsilon, \varepsilon])\right)=1\right)$ for all $n \geqq k$;

(5) fuzzy almost uniformly in an F-state $m$ (in a measure $P$ ), if, for every $\varepsilon>0$ and $\delta>0$ there are $a \in M(A \in K(M))$ such that $m(a)<\delta(P(A)<\delta)$ and an integer $k$ such that $a^{\perp} \leqq_{F}\left(x-x_{n}\right)([-\varepsilon, \varepsilon])\left(A^{c} \subset_{F}\left(f-f_{n}\right)^{-1}([-\varepsilon, \varepsilon])\right)$ for all $n \geqq k$;

(6) in an F-state $m($ in a measure $P)$, if, for every $\varepsilon>0 \lim m\left(\left(x-x_{n}\right)([-\varepsilon, \varepsilon])\right)=$ $=1\left(\lim _{n \rightarrow \infty} P\left(\left(f-f_{n}\right)^{-1}([-\varepsilon, \varepsilon])\right)=1\right)$;

(7) in mean $p$, where $1 \leqq p<\infty$, if $\lim _{n \rightarrow \infty} \int\left|x-x_{n}\right|^{p} \mathrm{~d} m=0 \quad\left(\lim _{n \rightarrow \infty} \int_{\Omega}\left|f-f_{n}\right|^{p} \mathrm{~d} P=0\right)$.

We say that a sequence $\left\{x_{n}\right\}_{n \in N} \subset O(M)\left(\left\{f_{n}\right\}_{n \in N} \subset F(M)\right)$ is

(8) fuzzy fundamental everywhere, if, for every $\varepsilon>0$,

$$
\begin{aligned}
& \bigcup_{k=1}^{\infty} \bigcap_{n, s=k}^{\infty}\left(x_{n}-x_{s}\right)([-\varepsilon, \varepsilon])={ }_{F} 1 \\
& \left.\bigcup_{k=1}^{\infty} \bigcap_{n, s=k}^{\infty}\left(f_{n}-f_{s}\right)^{-1}([-\varepsilon, \varepsilon])={ }_{F} \Omega\right) ;
\end{aligned}
$$

(9) fundamental almost everywhere in an F-state $m$ (in a measure P), if, for every $\varepsilon>0$,

$$
\begin{aligned}
& m\left(\bigcup_{k=1}^{\infty} \bigcap_{n, s=k}^{\infty}\left(x_{n}-x_{s}\right)([-\varepsilon, \varepsilon])\right)=1 \\
& \left(P\left(\bigcup_{k=1}^{\infty} \bigcap_{n, s=k}^{\infty}\left(f_{n}-f_{s}\right)^{-1}([-\varepsilon, \varepsilon])\right)=1\right) ;
\end{aligned}
$$

(10) fuzzy fundamental uniform, if, for every $\varepsilon>0$, there is an integer $k$ such that $\left(x_{n}-x_{s}\right)([-\varepsilon, \varepsilon])={ }_{F} 1$ $\left(\left(f_{n}-f_{s}\right)^{-1}([-\varepsilon, \varepsilon])={ }_{F} \Omega\right)$ for all $n, s \geqq k ;$

(11) fundamental uniform almost everywhere in an F-state $m$ (in a measure $P$ ), if, for every $\varepsilon>0$, there is an integer $k$ such that $m\left(\left(x_{n}-x_{s}\right)([-\varepsilon, \varepsilon])\right)=1$ $\left(P\left(\left(f_{n}-f_{s}\right)^{-1}([-\varepsilon, \varepsilon])\right)=1\right)$ for all $n, s \geqq k$; 
(12) fuzzy fundamental almost uniform in an F-state $m$ (in a measure $P$ ), if, for every $\delta>0$, there is an $a \in M(A \in K(M))$ such that $m(a)<\delta(P(A)<\delta)$, and for every $\varepsilon>0$ there is an integer $k$ such that

$$
a^{\perp} \leqq_{F}\left(x_{n}-x_{s}\right)([-\varepsilon, \varepsilon])\left(A^{c} \subset_{F}\left(f_{n}-f_{s}\right)^{-1}([-\varepsilon, \varepsilon])\right) \text { for all } n, s \geqq k \text {; }
$$

(13) fundamental in an $F$-state $m$ (in a measure $P$ ), if, for every $\varepsilon>0$,

$$
\begin{aligned}
& \left.\lim _{n, s \rightarrow \infty} m\left(x_{n}-x_{s}\right)([-\varepsilon, \varepsilon])\right)=1 \\
& \left(\lim _{n, s \rightarrow \infty} P\left(\left(f_{n}-f_{s}\right)^{-1}([-\varepsilon, \varepsilon])\right)=1\right) ;
\end{aligned}
$$

(14) fundamental in mean $p$, where $1 \leqq p<\infty$, if

$$
\lim _{n, s \rightarrow \infty} \int\left|x_{n}-x_{s}\right|^{p} \mathrm{~d} m=0\left(\lim _{n, s \rightarrow \infty} \int_{\Omega}\left|f_{n}-f_{s}\right|^{p} \mathrm{~d} P=0\right) .
$$

Theorem 3.2. Let $x, x_{n} \in O(M)$ and $f, f_{n} \in F(M)$, for any $n \geqq 1$, be such that $x \sim f, x_{n} \sim f_{n}, n \geqq 1$. The sequence $\left\{x_{n}\right\}_{n \in N}$ converges to $x$ in an arbitrary sense from (1) through (14) if and only if the sequence $\left\{f_{n}\right\}_{n \in N}$ converges to $f$ in the corresponding sense.

Proof. Suppose that the sequence $\left\{x_{n}\right\}_{n \in N}$ converges to $x$ fuzzy everywhere. Let $\varepsilon>0$ and denote

$$
a=\bigcup_{k=1}^{\infty} \bigcap_{n=k}^{\infty}\left(x-x_{n}\right)([-\varepsilon, \varepsilon]) \text { and } A=\bigcup_{k=1}^{\infty} \bigcap_{n=k}^{\infty}\left(f-f_{n}\right)^{-1}([-\varepsilon, \varepsilon]) .
$$

Then $a \in M$ and $A \in K(M)$, and by Lemma 1.2 they fulfil the condition (1.3). By the assumption we have $a={ }_{F} 1$, hence there is a $c \in W_{1}(M)$ such that $a^{\perp} \cap c \leqq 1 / 2$, which is equivalent to $\left\{a^{\perp}>1 / 2\right\} \subset\{c=1 / 2\}$. Then $\left\{a^{\perp}>1 / 2\right\} \subset A^{c} \subset$ $\subset\left\{a^{\perp} \geqq 1 / 2\right\}=\left\{a^{\perp}>1 / 2\right\} \cup\left\{a^{\perp}=1 / 2\right\} \subset\{c=1 / 2\} \cup\left\{a \cup a^{\perp}=1 / 2\right\}=$ $=\left\{c \cap\left(a \cup a^{\perp}\right)=1 / 2\right\}$. Put $d=c \cap\left(a \cup a^{\perp}\right)$, then evidently $d \in W_{1}(M)$ and $A \cap \Omega^{c}=\emptyset \subset\{d=1 / 2\}$ and $A^{c} \cap \Omega=A^{c} \subset\{d=1 / 2\}$, too, therefore $A \Delta \Omega \subset$ $\subset\{d=1 / 2\}$, which implies $A={ }_{F} \Omega$ i.e. the sequence $\left\{f_{n}\right\}_{n \in N}$ converges to $f$ fuzzy everywhere.

Now we prove the converse assertion. Suppose that $A={ }_{F} \Omega$ where $A=$ $=\bigcup_{k=1}^{\infty} \bigcap_{n=k}^{\infty}\left(f-f_{n}\right)^{-1}([-\varepsilon, \varepsilon])$ for some $\varepsilon>0$. Then there is a $c \in W_{1}(M)$ such that $A^{c}=A^{c} \cap \Omega \subset\{c=1 / 2\}$. In view of the above, we have $x_{a} \sim I_{A}$ where $a=$ $=\bigcup_{k=1}^{\infty} \bigcap_{n=k}^{\infty}\left(x-x_{n}\right)([-\varepsilon, \varepsilon])$. Therefore $\left\{a^{\perp}>1 / 2\right\} \subset A^{c} \subset\{c=1 / 2\}$, which gives $a^{\perp} \cap c \leqq 1 / 2$ or $a={ }_{F} 1$.

Other types of convergences may be proved in an analogous way. 
Proposition 3.3. If a sequence $\left\{x_{n}\right\}_{n \in N} \subset O(M)$ converges to $x \in O(M)$ fuzzy uniformly then the sequence $\left\{x_{n}\right\}_{n \in N}$ is fuzzy fundamental uniform. Conversely, if $\left\{x_{n}\right\}_{n \in N}$ is fuzzy fundamental uniform, then there is an $x \in O(M)$ such that $\left\{x_{n}\right\}_{n \in N}$ converges to $x$ fuzzy uniformly.

Proof. Let $f, f_{n} \in F(M)$ for any $n \geqq 1$ be such that $x \sim f$ and $x_{n} \sim f_{n}$. By Theorem 3.2 the sequence $\left\{f_{n}\right\}_{n \in N}$ converges to $f$ fuzzy uniformly. For every $\varepsilon>0$ there is an integer $k$ such that $\left(f-f_{n}\right)^{-1}([-\varepsilon, \varepsilon])={ }_{F} \Omega$ for all $n \geqq k$. By Lemma 2.4 there is a $c \in W_{1}(M)$ such that $\left(f-f_{n}\right)^{-1}\left([-\varepsilon, \varepsilon]^{c}\right) \subset\{c=1 / 2\}$ for every $\varepsilon>0$ and for all $n \geqq k$. Denote $A=\Omega-\{c=1 / 2\}$. It is evident that $A \in K(M)$ and $A={ }_{F} \Omega$. The sequence $\left\{f_{n}\right\}_{n \in N}$ converges to $f$ uniformly on the set $A$, which is equivalent to the assertion that, for every $\varepsilon>0$, there is an integer $k=k(\varepsilon)$ such that for all $n, s \geqq k, \Omega={ }_{F} A \subset\left(f_{s}-f_{n}\right)^{-1}([-\varepsilon, \varepsilon])$. This gives that the sequence $\left\{f_{n}\right\}_{n \in N}$ is fuzzy fundamental uniform and applying Theorem 3.2, we obtain that the sequence $\left\{x_{n}\right\}_{n \in N}$ is fuzzy fundamental uniform, too.

Suppose now that $\left\{x_{n}\right\}_{n \in N}$ is fuzzy fundamental uniform. Due to Theorem 3.2, $\left\{f_{n}\right\}_{n \in N}$ is fuzzy fundamental uniform. Hence, for any integer $i \geqq 1$ there is an integer $k=k(i)$ such that $A_{n, s, i}:=\left(f_{n}-f_{s}\right)^{-1}([-1 / i, 1 / i])={ }_{F} \Omega$ for any $n, s \geqq k$. Put $A_{0}=\bigcap_{i=1}^{\infty} \bigcap_{n, s=k(i)}^{\infty} A_{n, s, i}$. Then $A_{0} \in K(M)$ and $A_{0}={ }_{F} \Omega$. For any $\varepsilon>0$ we find an integer $i$ such that $\varepsilon>1 / i>0$, which entails that for any $n, s \geqq k(i)$ we have $\Omega={ }_{F} A_{0} \subset\left(f_{n}-f_{s}\right)^{-1}([-\varepsilon, \varepsilon])$, in other words, $\left\{f_{n}\right\}_{n \in N}$ is fundamental uniform on $A_{0}$. In view of a classical result, there is a $K(M)$-measurable function $f$ such that $\left\{f_{n}\right\}_{n \in N}$ converges uniformly to $f$ on $A_{0}$, that is $\left\{f_{n}\right\}_{n \in N}$ converges to $f$ fuzzy uniformly, too. According to the Theorem 1.3, there is an $F$-observable $x$ such that $x \sim f$, which in view of Theorem 3.2 yields that $\left\{x_{n}\right\}_{n \in N}$ converges fuzzy uniformly to $x$.

Analogously we can prove similar results on the relationship of the fundamentality of a given type of convergence and the existence of the limit-observable of the given type for $F$-observables.

It is clear that:

(i) the convergence (fundamental) almost everywhere follows from the convergence fuzzy (fundamental) everywhere, as well as from the convergence (fundamental) uniform almost everywhere;

(ii) the convergences fuzzy (fundamental) everywhere, (fundamental) uniform almost everywhere and fuzzy (fundamental) almost uniform follow from the convergence fuzzy (fundamental) uniform;

(iii) the convergence (fundamental) in an $F$-state $m$ (in a measure $P$ ) follows from the convergence (fundamental) uniform almost everywhere;

(iv) the convergence fuzzy (fundamental) almost uniform follows from the convergence (fundamental) uniform almost everywhere. 
Theorem 3.4. Let $x, x_{n} \in O(M)$ for any $n \in N$. The following statements are equivalent:

(i) A sequence $\left\{x_{n}\right\}_{n \in N}$ converges to $x$ fuzzy almost uniformly in an F-state $m$.

(ii) A sequence $\left\{x_{n}\right\}_{n \in N}$ converges to $x$ almost everywhere in an F-state $m$.

Proof. Suppose (i). Let $\varepsilon>0$ and put $\delta=1 / i, i=1,2, \ldots$ Then there is an $a_{i} \in M$ such that $m\left(a_{i}\right)<1 / i$ and there is an integer $k=k(i)$ such that $a_{i}^{1} \leqq_{F}$ $\leqq_{F}\left(x-x_{n}\right)([-\varepsilon, \varepsilon])$ for all $n \geqq k$, as well as $a_{i}^{\perp} \leqq_{F} \bigcap_{n=k}^{\infty}\left(x-x_{n}\right)([-\varepsilon, \varepsilon])$. Put $a=\bigcap a_{i}$. We have $0 \leqq m(a) \leqq m\left(a_{i}\right)<1 / i$ for $i=1,2, \ldots$, which gives $m(a)=0$ or $m\left(a^{\perp}\right)=1$. Then $a^{\perp}=\bigcup_{i=1}^{\infty} a_{i}^{\perp} \leqq \bigcup_{i=1}^{\infty} \bigcap_{n=k}^{\infty}\left(x-x_{n}\right)([-\varepsilon, \varepsilon]) \leqq \bigcup_{j=1}^{\infty} \bigcap_{n=j}^{\infty}\left(x-x_{n}\right)$. . $([\varepsilon, \varepsilon])$, which implies $1=m\left(a^{\perp}\right) \leqq m\left(\bigcup_{j=1}^{\infty} \bigcap_{n=j}^{\infty}\left(x-x_{n}\right)([-\varepsilon, \varepsilon]) \leqq 1\right.$.

Let now (ii) hold. By Theorem 3.2, a sequence $\left\{f_{n}\right\}_{n \in N}$ converges to $f$ almost everywhere in a measure $P$, where $f, f_{n} \in F(M)$ are such that $x \sim f, x_{n} \sim f_{n}$ for any $n \in N$, and by the Jegorov theorem [9], the sequence $\left\{f_{n}\right\}_{n \in N}$ converges to $f$ almost uniformly in a measure $P$, which gives that this sequence converges to $f$ fuzzy almost uniformly in a measure $P$, too, and again applying Theorem 3.2 we obtain that the sequence $\left\{x_{n}\right\}_{n \in N}$ converges to $x$ fuzzy almost uniformly in an F-state $m$.

Proposition 3.5. Let a sequence $\left\{x_{n}\right\}_{n \in N} \subset O(M)$ converge to $x \in O(M)$ in an $F$-state $m$. Then this sequence is fundamental in the F-state $m$.

Proof. Let $\varepsilon>0$ and let $x \sim f, x_{n} \sim f_{n}$ for all $n \geqq 1$, where $f, f_{n} \in F(M)$. By the assumption and by Theorem 3.2 we have

$$
\begin{aligned}
& \lim _{n \rightarrow \infty} P\left(\left(f-f_{n}\right)^{-1}([-\varepsilon / 2, \varepsilon / 2])\right)=1 \text { or } \\
& \lim _{n \rightarrow \infty} P\left(\left(f-f_{n}\right)^{-1}\left([-\varepsilon / 2, \varepsilon / 2]^{c}\right)\right)=0 .
\end{aligned}
$$

Because $\left(f_{n}-f_{s}\right)^{-1}\left([-\varepsilon, \varepsilon]^{c}\right) \subset\left(f_{n}-f\right)^{-1}\left([-\varepsilon / 2, \varepsilon / 2]^{c}\right) \cup$ $\cup\left(f-f_{s}\right)^{-1}\left([-\varepsilon / 2, \varepsilon / 2]^{c}\right)$, we have $P\left(\left(f_{n}-f_{s}\right)^{-1}\left([-\varepsilon, \varepsilon]^{c}\right)\right) \leqq$ $\leqq P\left(\left(f_{n}-f\right)^{-1}\left([-\varepsilon / 2, \varepsilon / 2]^{c}\right)\right)+P\left(\left(f-f_{s}\right)^{-1}\left([-\varepsilon / 2, \varepsilon / 2]^{c}\right)\right)$ and hence $0 \leqq$ $\leqq \lim _{n, s \rightarrow \infty} P\left(\left(f_{n}-f_{s}\right)^{-1}\left([-\varepsilon, \varepsilon]^{c}\right)\right) \leqq 0$, which gives $\lim _{n, s \rightarrow \infty} P\left(\left(f_{n}-f_{s}\right)^{-1}([-\varepsilon, \varepsilon])\right)=1$.

Proposition 3.6. If a sequence $\left\{x_{n}\right\}_{n \in N} \subset O(M)$ is fundamental in an F-state $m$, then there is an F-observable $x$ such that the sequence $\left\{x_{n}\right\}_{n \in N}$ converges to $x$ in the F-state $m$.

Proof. Let $\left\{f_{n}\right\}_{n \in N} \subset F(M)$ be such that $x_{n} \sim f_{n}$ for all $n \in N$. By Theorem 3.2 the sequence $\left\{f_{n}\right\}_{n \in N}$ is fundamental in a measure $P$ and by Theorem 6.44 in [9] 
there is a $K(M)$-measurable function $f$ such that the sequence $\left\{f_{n}\right\}_{n \in N}$ convergers to $f$ in the measure $P$. According to Theorem 1.3, there is an $F$-observable $x$ such that $x \sim f$ and by Theorem 3.2 the sequence $\left\{x_{n}\right\}_{n \in N}$ converges to $x$ in the $F$-state $m$.

\title{
References
}

[1] S. P. Gudder, H. C. Mullikin: Measure theoretic convergences of observables and operators. Journal of Mathematical Physics, 14 (1973), 234-242.

[2] B. Riečan: A new approach to some notions of statistical quantum mechanics. Busefal, 35, (1988), 4-6.

[3] A. Dvurečenskij: On existence of probability measures on fuzzy measurable spaces (to appear in Fuzzy Sets and Systems).

[4] K. Piasecki: Probability of fuzzy events defined as denumerable addivity measure. Fuzzy Sets and Systems, 17 (1985), 271-284.

[5] A. Dvurečenskij, A. Tirpáková: A note on a sum of observables in $F$-quantum spaces and its applications. Busefal, 35 (1988), 132-137.

[6] $K$. Piasecki: On fuzzy $P$-measures. In: Proc. First Winter School on Measure Theory, Liptovský Ján, Jan. 10-15, 1988, 108-112.

[7] A. Dvurečenskij: On a representation of observables in fuzzy measurable spaces (to appear in J. Math. Anal. Appl.).

[8] A. Dvurečenskij, B. Riečan: On joint distribution of observables for $F$-quantum spaces (to appear in Fuzzy Sets and Systems).

[9] T. Neubrunn, B. Riečan: Measure and Integral (Slovak). VEDA Bratislava 1981.

Súhrn

\section{FUZZY ROVNOSŤ A KONVERGENCIE $F$-POZOROVATELNÝCH V F-KVANTOVÝCH PRIESTOROCH}

\author{
Ferdinand Chovanec, František KôpKa
}

V F-kvantovom priestore zavedením relácií fuzzy rovnosti a fuzzy nerovnosti sa definuji rôzne typy konvergencii pre postupnosti $F$-pozorovatelných a využitím reprezentácie $F$-pozorovatelných bodovými funkciami definovanými vo vhodnom meratelnom priestore sa dokážu niektoré konvergenčné vety.

Authors' address: RNDr. Ferdinand Chovanec, RNDr. František Kôpka, Katedra matematiky VVTŠ, 03119 Liptovský Mikuláš. 